\title{
One-year Observational Study of an Iris Camera Corneal Opacification Measurement and Pentacam Densitometry in Keratoconus after Corneal Crosslinking
}

Chimdi Emma-Duru ( $\square$ chimdi.emma-duru@postgrad.manchester.ac.uk)

University of Manchester

Fiona Carley

Manchester Royal Eye Hospital

Chantal Hillarby

University of Manchester

Tariq Aslam

Manchester Royal Eye Hospital

\section{Research Article}

Keywords: Collagen crosslinking, Keratoconus, iris camera, corneal opacification measure, corneal imaging

Posted Date: May 12th, 2021

DOI: https://doi.org/10.21203/rs.3.rs-492998/v1

License: (c) (1) This work is licensed under a Creative Commons Attribution 4.0 International License.

Read Full License 


\section{Abstract}

Purpose: To investigate levels of corneal haze in adult patients with keratoconus treated by corneal crosslinking, using Iris camera and Corneal densitometry imaging.

Methods: This was a prospective, observational cross-sectional study on patients attending the Manchester Royal Eye Hospital outpatient department. Iris camera, Pentacam densitometry and visual acuity measurements were collected from patients in two groups. In group A, patients were assessed before and after crosslinking $(\mathrm{CXL})$, whereas in group $\mathrm{B}$, measurements were made for an extended period after their $\mathrm{CXL}$ had been performed.

Results: Group A consisted of 13 eyes of 13 patients, and group B consisted of 41 eyes of 41 patients. In group A average age was 26.69 (7.19) years. Average time of measurements taken before CXL was 1.85 (0.80) months and after CXL 12.69 (0.63) months. When comparing values before $C X L$ and after, there was a significantly decreased COM score $(P=0.001)$ and Pentacam Corneal densitometry measurement $(P=0.003)$ representing improved corneal clarity. There was a corresponding significant improvement in vision in all eyes in group A after CXL procedure, and $100 \%$ of eyes reached a BCVA of $\leq 20 / 50(\leq 0.4$ LogMAR) ( $P=0.002)$. In group $B$, the mean first examination time was post-treatment 3.24 (1.48) months and for the second examination, post-treatment $12.56(0.81)$ months. Between these two visits, there was a significant decrease in COM score $(P=0.002)$ and corneal densitometry $(P=0.0004)$ as well as improvement in BCVA ( $P=0.0002)$. In this group also, $100 \%$ of eyes reached a BCVA of $\leq 20 / 50(\leq 0.4$ LogMAR) by the time of their last follow up.

Conclusion: There is a significant improvement in corneal opacification measures using both Iris camera and densitometry when assessing before and after CXL and also sequentially 3 and 12 months after the procedure. The iris camera represents a promising, portable and practical means of assessing and monitoring corneal clarity for this purpose.

\section{Key Messages}

1. Many prior clinical studies have demonstrated the efficacy of corneal crosslinking as a treatment for keratoconus.

2. This study demonstrates that both the Iris Camera and Pentacam can be used to objectively determine changes in corneal transparency following corneal crosslinking.

3. The iris camera technique particularly shows potential as a non invasive, portable tool for such assessments.

\section{Introduction}


Keratoconus (KCN) is a spontaneous and sometimes asymmetric ectatic degeneration of the cornea characterised by a progressive thinning and an eventual steepening and protrusion of the thinned corneal surface giving it the shape of a cone or a dome [1]. Consequences of this corneal degeneration include high myopia and irregular astigmatism which, when combined with thinning leads to poor vision [2].

$\mathrm{KCN}$ is progressive involving structural changes at a molecular level. The management of KCN is dependent on the level of severity or the rate of advancement [3]. At an early stage, optical corrections using spectacles and contact lenses (rigid contact lens) are typically employed [4]. Corneal transplantation is indicated in advanced cases of keratoconus where refractive corrections prove insufficient at managing severe irregular astigmatism or in patients intolerant to contact lens use [5]. Surgery may also be indicated for scarring or following an episode of corneal hydrops (rupture of the Descemet's membrane).

An alternative treatment for several forms of corneal ectasia that has emerged over the last two decades is corneal crosslinking (CXL). Procedures inducing crosslinks have been identified as a limiting factor to disease progression. Corneal crosslinking was first performed in 1998 at the University Eye Clinic in Dresden, Germany $[6,7]$. This promising, minimally invasive, procedure has become an established treatment for progressive keratoconus - designed to slow down or halt its progression, thereby reducing the requirement for a transplant [8]. Over the years, this procedure has been performed predominantly on adults; however, recent findings suggest that it has been extended to paediatric populations ( $<18 y e a r s)$ [9, 10]. Corneal crosslinking works by strengthening and stabilising the corneal stroma's collagen lamellae by creating new molecular chemical bonds (cross-links) through a process known as localised photopolymerisation $[11,12]$. This treatment involves using riboflavin (Vitamin B2) and ultraviolet radiation (UVA) using an irradiation device that emits radiation between 360-380 microns in homologous sequences in fusion with the riboflavin.

The procedure is typically performed using topical anaesthetic and entails 6-7mm diameter of the central epithelial cell layer being debrided under sterile conditions using a blunt spatula. The debrided area is then treated with $0.1 \%$ riboflavin drops frequently for a total of 25 minutes to ensure the full penetration and saturation of the corneal stroma. The saturated cornea is then exposed to the UVA with the riboflavin acting as a synthesiser with an absorption peak of approximately 370 microns which produces reactive oxygen that eventually forms the covalent bonds within the stroma thereby making the cornea stiffer.

There are two types of crosslinking procedures: the standard (Dresden) and the accelerated protocol. The standard protocol is the most conventional crosslinking techniques using $3 \mathrm{~mW} / \mathrm{cm}^{2} \mathrm{UV}$ intensity and 30 minute radiation $[4,7]$. Although the standard Dresden protocol is effective at treating Keratoconus, its major drawback was the duration it took for the procedure [13]. The Accelerated protocol emerged as an alternative technique to decrease the treatment time and employed the Bunson-Roscoe law which stated that increasing the radiative dose with a constant exposure of $5.47 \mathrm{~J} / \mathrm{cm}^{2}$ produced the same treatment effect [14]. In the accelerated procedure however, the cornea is radiated using $9 \mathrm{~mW} / \mathrm{cm}^{2} \mathrm{UV}$ intensity for 10 minutes $[13,15]$. After crosslinking, patients are examined post-operatively to ensure reepithelialization with subsequent follow up to assess response to treatment. 
Over recent years, the need for objective quantification of corneal haze as a baseline for disease severity has increased significantly [16]. The severity of a disease or the corneal haze levels was previously categorised subjectively by a clinical observer as either healthy, mild, moderate or severely clouded based on the anterior chamber's visibility during clinical assessment. However, this individual evaluation is subject to bias and could lead to discrepancies in disease grading and classification [17]. The iris camera has been used to produce reliable images of the cornea analysed using purpose-built algorithms to determine a corneal opacification measure (COM). The algorithms involve a process of removing flash artefact and determining the apparent level of penetrance of the infra-red light used to produce the image [16].

An alternative system of objective corneal clarity assessment involves Pentacam densitometry - this is a system that employs a rotating Scheimpflug camera to give a 3-dimensional cross-section of the cornea as other optic media $([17,18]$.

The purpose of this study was to evaluate corneal clarity in patients with keratoconus and how this is affected by corneal crosslinking (CXL). Measurements were taken using the iris camera and Pentacam in addition to best-corrected visual acuity.

At each appointment the patients best corrected visual acuity (BCVA) in LogMAR, was obtained via clinical notes as well as pertinent clinical history.

\section{Participants And Methods}

This was a prospective, observational study. The study was approved by the Manchester University NHS Foundation Trust, Manchester, UK, NREC local ethics committee. All aspects of the research comply with the tenets of the Declaration of Helsinki. Participants were recruited from the Corneal Ophthalmology clinics at the Manchester Royal Eye Hospital (MREH) and informed consent obtained from all participants. Patients were only assessed at existing appointments made for clinical reasons, and imaging was restricted to these occasions. Inclusion criteria were patients with keratoconus over the age of 18 years, with no other ophthalmic diagnosis, who had either already undergone corneal crosslinking or who were due to have this procedure. Patients who were recruited belonged to two distinct groups. Group A included patients who were able to be examined before and after corneal cross-linking. Patients in Group B had already undergone corneal cross-linking and so measurements were taken post-treatment at 3 and 12 months.

The accelerated protocol was carried out for all patients in both groups [19]. Benoxinate $0.4 \%$ was used to anaesthetize the cornea before debridement. For corneal debridement, $20 \%$ of alcohol was applied for 20 seconds on the cornea with both lids separated by a speculum. The central corneal thickness (CCT) was assessed using Ultrasound pachymetry following debridement of the epithelial layer and recorded. The application of one drop of $0.4 \%$ hypotonic riboflavin to the cornea was made when the CCT was greater than $400 \mu \mathrm{m}$. This process was carried out for 9 minutes (i.e., a drop every minute). Ultra-violet-A (9.86Mw-UVA) was used to irradiate the central cornea for 9 minutes (UVX 1000 system; IROC Innocross 
AG, Zurich, Switzerland) with pachymetry measurements performed every 3 minutes. Once the procedure was completed, topical antibiotics and dexamethasone $1 \%$ were instilled in the cross-linked eye and pain relief medications for 4-8 weeks post-treatment.

Imaging taken included corneal opacification measure (COM) score from an iris recognition camera described in detail in previous publications [16]. The iris camera was used to obtain corneal images at each visit with standard lighting and no pharmacological dilation. Images were subject to analysis to obtain the COM score. Also, corneal densitometry measurements were obtained from an Oculus Pentacam. Patients with an inability to fixate on the iris camera during image testing and capture were excluded as were patients unable to perform Scheimpflug examinations or return for review appointments. At each appointment the patient's best-corrected visual acuity (BCVA) in LogMAR was obtained via clinical notes together with any pertinent clinical history.

Only one eye of each patient was used for the study. Corneal haze in keratoconus may affect each eye differently and, in both groups, some patients had corneal crosslinking done on both eyes. For this study, if both eyes had undergone crosslinking, we chose the eye with the worse level of COM score for analysis.

We described the COM score and corneal densitometry values over time. For group A, data was taken at baseline (before crosslinking) and at 12 months after crosslinking. For group B the same measures were assessed approximately 3 months after crosslinking and then one year after that first measurement.

\section{Statistical Analysis}

Data analyses were preformed using IBM SPSS statistics for Mac, Version 25, NY: IBM Corp and GraphPad prism9 for Mac was used for statistical analysis. Shapiro-Wilks test was used to assess normality of data. Data were presented for each visit and follow-up as the mean \pm standard deviation (SD) at baseline (1 month and 3 months) and at 12 months from initial visit. Box and whisker plot was used to compare baseline and follow-up visits for both groups. Paired Wilcoxon-signed rank test was used to analyse follow-ups as compared to baseline. A p-value $<0.05$ was considered statistically significant.

\section{Results}

\section{Group A}

Patient baseline characteristics are presented in table 1. including details of patient visit times. There were 13 eyes analysed in group A and 41 eyes in group B. The mean pre and post-treatement meaurements are reported in table 2.

Group A represented patients in whom measurements were able to be taken before and approximately 12 months after crosslinking. Analysis of COM scores in this group indicated a statistically significant 
decrease in the levels of corneal haze at the 12 th month follow up ( $P=0.001$, Fig. I). A majority of these patients showed COM scores values between zero ( 0 ) and 0.5 at the follow-up visit.

The change in corneal densitometry measurements in group A also illustrated a marked decrease after 12 months from the day of treatment (Fig. II).

Comparing The mean best corrective visual acuity (BCVA) before and after crosslinking in group A showed statistically significant improvement $(P=0.002)$ with a majority of these patients achieving posttreatment BCVAs of 0.6 LogMAR (6/24) or better (Fig. III).

\section{Group B}

Group B represented patients in whom we were only able to obtain measurements around 3 then 12 months after crosslinking. Analysis of the COM score in group B showed a decline in the level of haze on the cornea between the first and second postoperative follow-up visits with a p-value of 0.002 (Fig. IV). Similarly, the corneal densitometry measurement (Fig. V) indicated a significant reduction in corneal haze levels $(P=0.0004)$.

Over the twelve months, the BCVAs in group B also changed (Fig. $\mathrm{VI})$, showing significant improvements at the follow-up visit $(P=0.0002)$ with most of these patients achieving BCVAs of 0.6 LogMAR $(6 / 24$ Snellen) or better. The post-treatment measurements of the COM score, densitometry and BCVA are summarised in table 2.

\section{Discussion}

Over the last 15 years, treatment modalities for keratoconus using corneal crosslinking have improved steadily as it becomes a procedure of choice to treat early to moderate cases in an attempt to halt disease progression. Corneal clarity generally decreases as keratoconus progresses, and it is usually the anterior layers of the cornea that are predominantly affected [20]. Although the intention behind crosslinking is to stop the progression of the disease and in some cases improve vision, the procedure itself can induce haze. [21]. This haze or cloudiness of the cornea stems from the damage to the keratocytes within the anterior stroma during the treatment process and usually diminishes within the first few months but has been known to last as long as 12 months [22].

A current method employed to quantify corneal clouding in keratoconus is the Pentacam densitometry programme, which uses a Scheimpflug imagery to assess light scatter in the eyes anterior segment giving quantifiable data as a marker for clouding or clarity [1]. In this study, we also use the iris camera for the first time for this condition. The iris camera utilises a different technique from the Pentacam to analyse and measures corneal haze. This system uses near-infrared light that penetrates and captures images of even a densely clouded cornea, and the adapted algorithm calculates the pixel intensity of the image while eliminating artefacts to produce the corneal opacification measure (COM) [23]. Additional studies 
have further validated the use of the iris camera as a means to objectively quantify corneal clouding and demonstrated its reliability and repeatability as a useful clinical tool to monitor disease change. [16] Our group previously employed the novel technique to evaluate Mucopolysaccharidoses (MPS); a heterogeneous lysosomal storage disease that often results in visual deterioration or impairment.

For this study, we were able to use the iris camera to demonstrate significant changes in corneal haze before and after CXL and that the haze continues to improve after CXL.

We were able to similarly demonstrate these changes with the standard Pentacam imaging but establishing sensitivity of the iris camera to this may provide advantages as it is designed to measure clinically valid opacification and is more portable, practical and affordable. The iris camera is also easier to use for examiners and from our experience easier for patients. It appears from our findings that values of change had higher values of significance than for the Pentacam comparing assessments before and after $\mathrm{CXL}$.

Although the haze levels in our study using the COM scores as a baseline were predominantly mild, these values still showed a decrease within 12 months and were similar to findings by other authors [24] indicating a natural tendency for haze to decline following treatment. The subtle changes in densitometry measurements concur with these findings and evidence the iris camera's ability to produce an objective measurement of corneal clouding $[16,25]$. Our results also showed significant differences and improvements in visual acuity of (-0.02 LogMAR) at the follow-up visits similar to findings by [26] demonstrating that vision improved. These changes in COM scores and corneal densitometry coinciding with improvement in vision are indicative that in our study corneal clouding after crosslinking eventually resolved with time $[3,27]$.

\section{Conclusion}

In this study, both the Iris camera and Pentacam successfully demonstrated a capacity to monitor treatment through change in corneal opacification in patients with Keratoconus undergoing CXL therapy, validating their use as a useful tool in a clinical setting. This is the first time in particular that the novel iris camera technique has been applied to provide an objective measurement of opacification changes over time in patients who have undergone corneal crosslinking. We demonstrate that the iris camera and its image analysis software enable objective quantification of haze in corneal diseases and could play an important role in evaluating, investigating, and monitoring corneal clouding in patients who have undergone treatment.

\section{Declarations}

\section{Acknowledgement:}

The authors thank the Manchester Royal Eye Hospital (corneal clinic) for supporting patient recruitment for this study. Part of the data was presented at ARVO 2019, Vancouver, Canada. 


\section{Compliance with Ethical Standards:}

All procedures performed in this study involving human participants were in accordance with the institution's ethical standards and national research committee and the 1964 Declaration of Helsinki and its later amendments or comparable ethical standards. This study was approved by the MHS Bioethics Committee of the University of Manchester (227987). Informed consent was obtained from all individual participants included in the study. This article does not contain any studies with animals performed by any of the authors.

\section{Disclosure:}

The authors report no conflicts of interest in this work.

\section{Funding:}

No funding was received for conducting this study.

\section{References}

1. Alzahrani K, Mofty H, Lin EY, Carley F, Brahma A, Morley D, et al. Corneal Imaging and Densitometry Measurements in Juvenile and Adult Keratoconus Patients to Evaluate Disease Progression and Treatment Effects After Corneal Cross-Linking. Clin Optom (Auckl). 2019;11:173-80. doi: 10.2147/OPTO.S226000.

2. Mofty H, Alzahrani K, Carley F, Harper S, Brahma A, Au L, et al. Evaluation of corneal symmetry after UV corneal crosslinking for keratoconus. Clin Ophthalmol. 2017;11:2043-9. doi: 10.2147/OPTH.S143511.

3. Subasinghe SK, Ogbuehi KC, Dias GJ. Current perspectives on corneal collagen crosslinking (CXL). Graefes Arch Clin Exp Ophthalmol. 2018;256(8):1363-84. doi: 10.1007/s00417-018-3966-0.

4. Deshmukh R, Hafezi F, Kymionis GD, Kling S, Shah R, Padmanabhan P, et al. Current concepts in crosslinking thin corneas. Indian J Ophthalmol. 2019;67(1):8-15. doi: 10.4103/ijo.IJO_1403_18.

5. Pron G, leraci L, Kaulback K, Medical Advisory Secretariat HaQO. Collagen cross-linking using riboflavin and ultraviolet-a for corneal thinning disorders: an evidence-based analysis. Ont Health Technol Assess Ser. 2011;11(5):1-89.

6. Raiskup-Wolf F, Hoyer A, Spoerl E, Pillunat LE. Collagen crosslinking with riboflavin and ultraviolet-A light in keratoconus: long-term results. J Cataract Refract Surg. 2008;34(5):796-801. doi:

10.1016/j.jcrs.2007.12.039.

7. Wollensak G, Spoerl E, Seiler T. Riboflavin/ultraviolet-a-induced collagen crosslinking for the treatment of keratoconus. Am J Ophthalmol. 2003;135(5):620-7. doi: 10.1016/s00029394(02)02220-1. 
8. Ashar JN, Vadavalli PK. Long-term results of riboflavin ultraviolet A corneal collagen cross-linking for Keratoconus in Italy: the Siena eye cross study. Am J Ophthalmol. 2010;150(4):588; author reply -9. doi: 10.1016/j.ajo.2010.06.032.

9. Vinciguerra P, Albé E, Frueh BE, Trazza S, Epstein D. Two-year corneal cross-linking results in patients younger than 18 years with documented progressive keratoconus. Am J Ophthalmol. 2012;154(3):520-6. doi: 10.1016/j.ajo.2012.03.020.

10. Caporossi A, Mazzotta C, Baiocchi S, Caporossi T, Denaro R, Balestrazzi A. Riboflavin-UVA-induced corneal collagen cross-linking in pediatric patients. Cornea. 2012;31(3):227-31. doi: 10.1097/ico.0b013e31822159f6.

11. Shalchi Z, Wang X, Nanavaty MA. Safety and efficacy of epithelium removal and transepithelial corneal collagen crosslinking for keratoconus. Eye (Lond). 2015;29(1):15-29. doi: 10.1038/eye.2014.230.

12. Hersh PS, Stulting RD, Muller D, Durrie DS, Rajpal RK, Group USCS. United States Multicenter Clinical Trial of Corneal Collagen Crosslinking for Keratoconus Treatment. Ophthalmology. 2017;124(9):1259-70. doi: 10.1016/j.ophtha.2017.03.052.

13. Choi M, Kim J, Kim EK, Seo KY, Kim TI. Comparison of the Conventional Dresden Protocol and Accelerated Protocol With Higher Ultraviolet Intensity in Corneal Collagen Cross-Linking for Keratoconus. Cornea. 2017;36(5):523-9. doi: 10.1097/ICO.0000000000001165.

14. Ng ALK, Chan TC, Cheng AC. Conventionalversusaccelerated corneal collagen cross-linking in the treatment of keratoconus. Clinical \& Experimental Ophthalmology. 2016;44(1):8-14. doi: $10.1111 /$ ceo.12571.

15. Sorkin N, Varssano D. Corneal Collagen Crosslinking: A Systematic Review. Ophthalmologica. 2014;232(1):10-27. doi: 10.1159/000357979.

16. Aslam TM, Shakir S, Wong J, Au L, Ashworth J. Use of iris recognition camera technology for the quantification of corneal opacification in mucopolysaccharidoses. $\mathrm{Br} \mathrm{J}$ Ophthalmol. 2012;96(12):1466-8. doi: 10.1136/bjophthalmol-2011-300996.

17. Elflein HM, Hofherr T, Berisha-Ramadani F, Weyer V, Lampe C, Beck M, et al. Measuring corneal clouding in patients suffering from mucopolysaccharidosis with the Pentacam densitometry programme. Br J Ophthalmol. 2013;97(7):829-33. doi: 10.1136/bjophthalmol-2012-302913.

18. Fujioka M, Nakamura M, Tatsumi Y, Kusuhara A, Maeda H, Negi A. Comparison of Pentacam Scheimpflug camera with ultrasound pachymetry and noncontact specular microscopy in measuring central corneal thickness. Curr Eye Res. 2007;32(2):89-94. doi: 10.1080/02713680601115010.

19. Kirgiz A, Eliacik M, Yildirim Y. Different accelerated corneal collagen cross-linking treatment modalities in progressive keratoconus. Eye and Vision. 2019;6(1). doi: 10.1186/s40662-019-0141-6.

20. Anayol MA, Sekeroglu MA, Ceran BB, Dogan M, Gunaydin S, Yilmazbas P. Quantitative assessment of corneal clarity in keratoconus: a case control study of corneal densitometry. Eur J Ophthalmol. 2016;26(1):18-23. doi: 10.5301/ejo.5000644. 
21. Koller T, Mrochen $M$, Seiler T. Complication and failure rates after corneal crosslinking. J Cataract Refract Surg. 2009;35(8):1358-62. doi: 10.1016/j.jcrs.2009.03.035.

22. Dhawan S, Rao K, Natrajan S. Complications of corneal collagen cross-linking. J Ophthalmol. 2011;2011:869015. doi: 10.1155/2011/869015.

23. Javed A, Aslam T, Ashworth J. Use of new imaging in detecting and monitoring ocular manifestations of the mucopolysaccharidoses. Acta Ophthalmol. 2016;94(8):e676-e82. doi: 10.1111/aos.13098.

24. Greenstein SA, Fry KL, Bhatt J, Hersh PS. Natural history of corneal haze after collagen crosslinking for keratoconus and corneal ectasia: Scheimpflug and biomicroscopic analysis. J Cataract Refract Surg. 2010;36(12):2105-14. doi: 10.1016/j.jcrs.2010.06.067.

25. Javed A, Aslam T, Jones SA, Ashworth J. Objective Quantification of Changes in Corneal Clouding Over Time in Patients With Mucopolysaccharidosis. Invest Ophthalmol Vis Sci. 2017;58(2):954-8. doi: $10.1167 /$ iovs.16-20647.

26. Chen X, Stojanovic A, Eidet JR, Utheim TP. Corneal collagen cross-linking (CXL) in thin corneas. Eye Vis (Lond). 2015;2:15. doi: 10.1186/s40662-015-0025-3.

27. Wittig-Silva $C$, Chan E, Islam FM, Wu T, Whiting M, Snibson GR. A randomized, controlled trial of corneal collagen cross-linking in progressive keratoconus: three-year results. Ophthalmology. 2014;121(4):812-21. doi: 10.1016/j.ophtha.2013.10.028.

\section{Tables}

Due to technical limitations, tables are only available as a download in the Supplemental Files section.

\section{Figures}




\section{Group A}

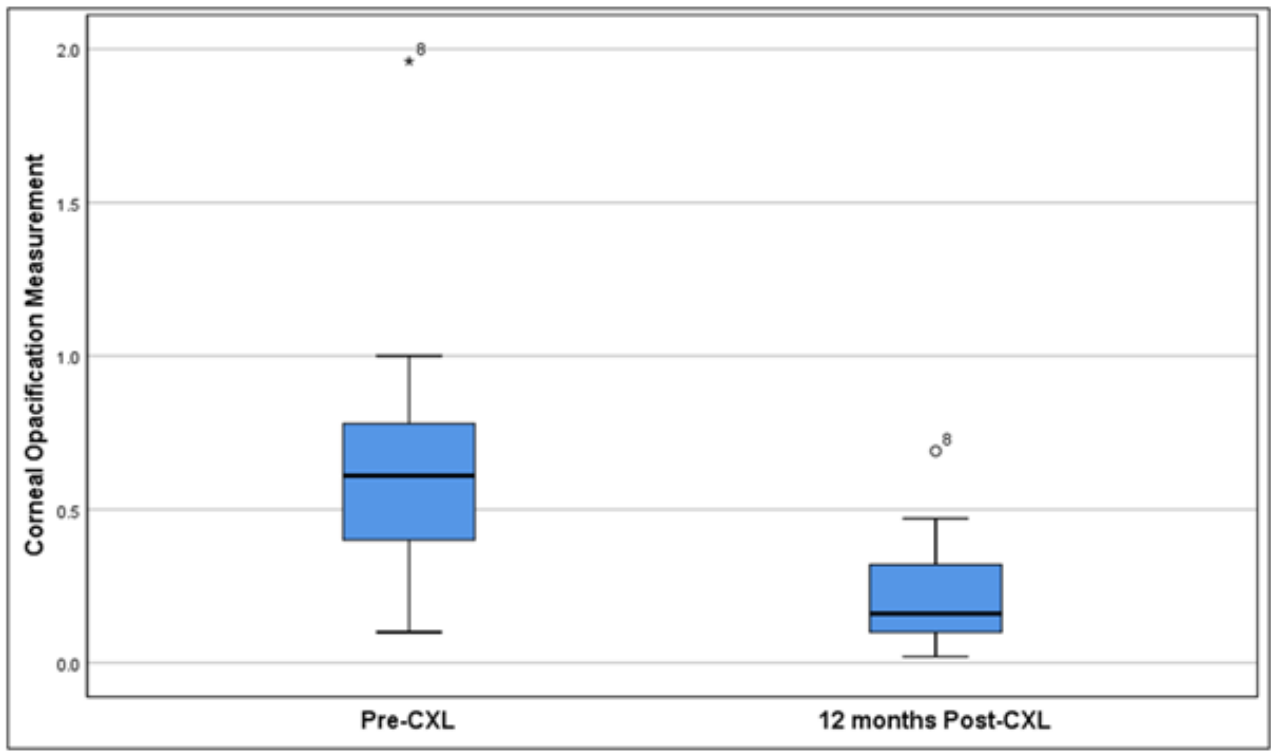

Figure 1

Iris Camera COM score before and after corneal crosslinking

\section{Group A}

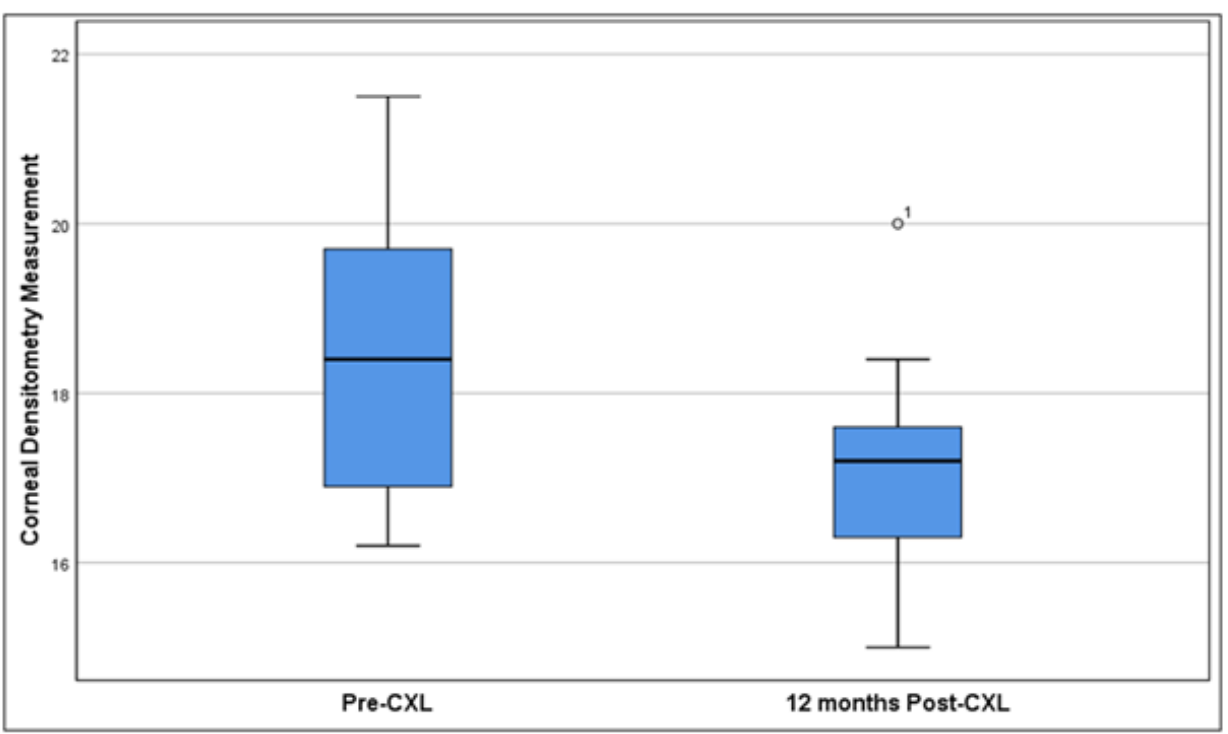


Figure 2

Corneal Densitometry before and after corneal crosslinking

\section{Group A}

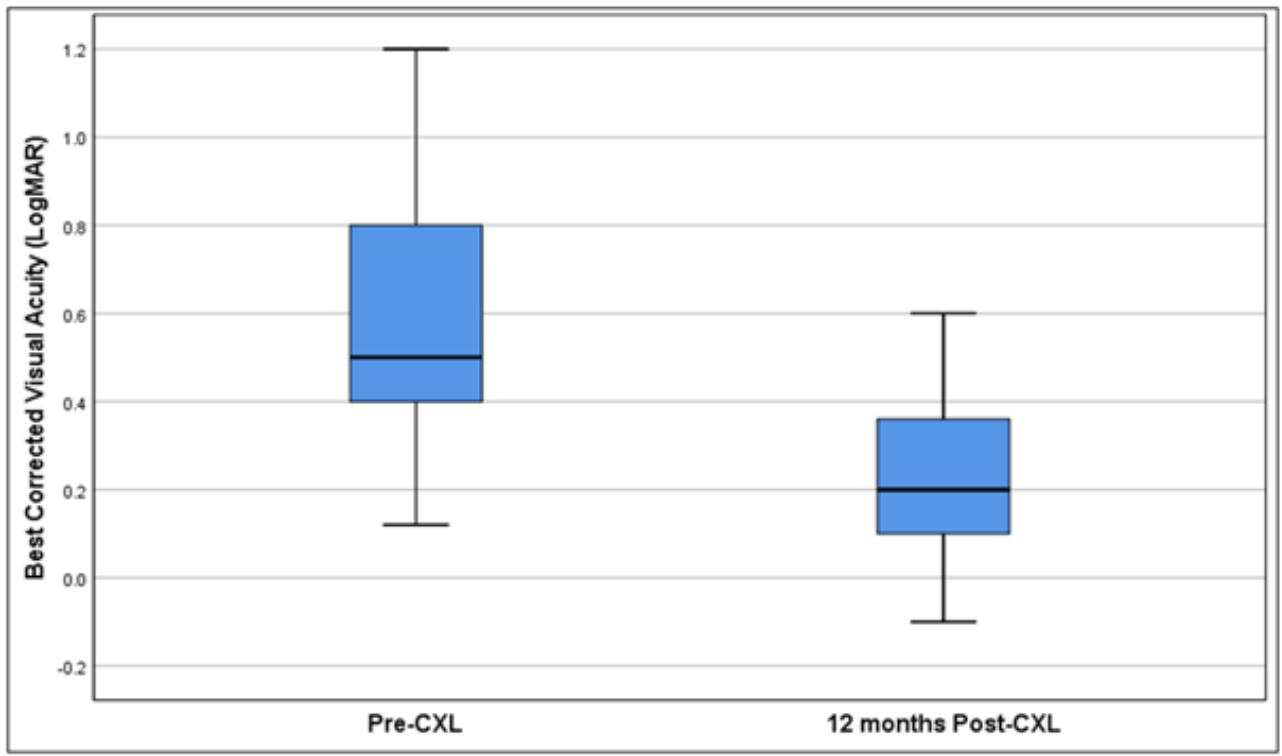

Figure 3

BCVA before and after corneal crosslinking 


\section{Group B}

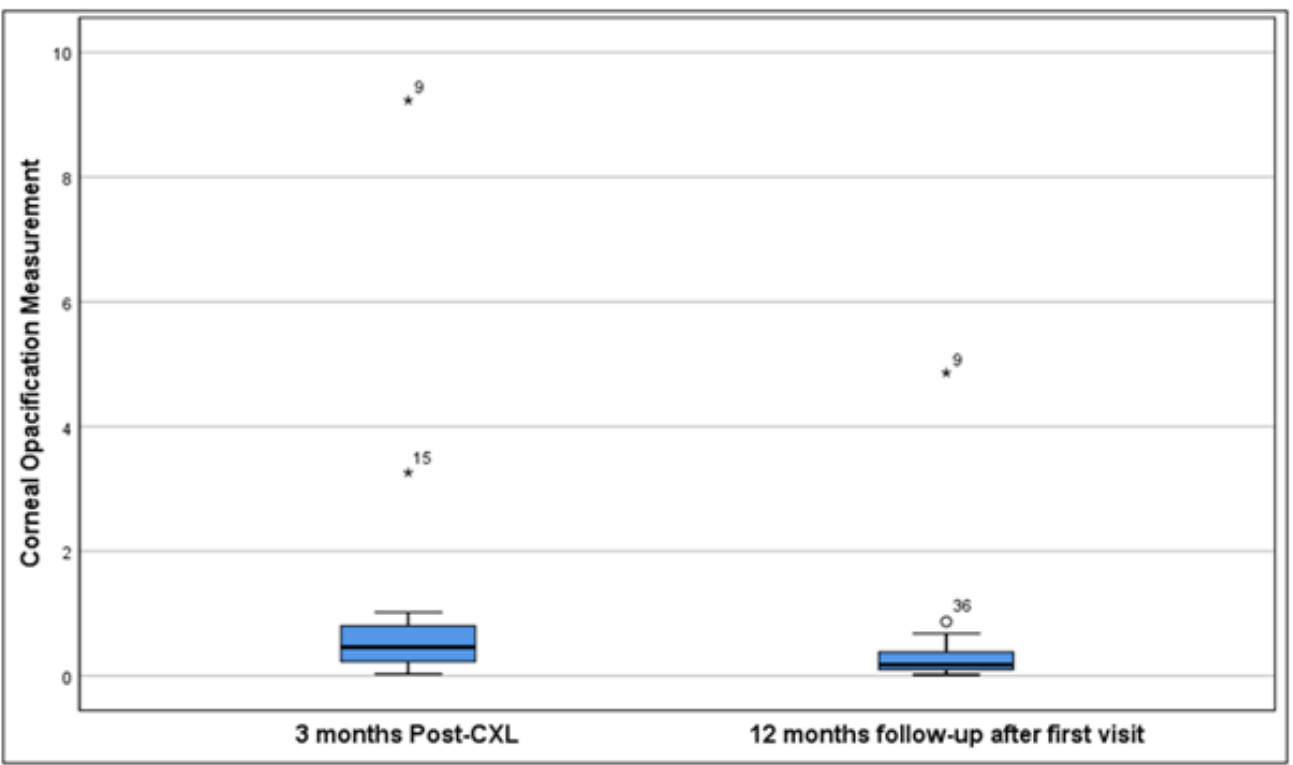

Figure 4

Iris Camera COM scores on two occasion post-crosslinking

\section{Group B}

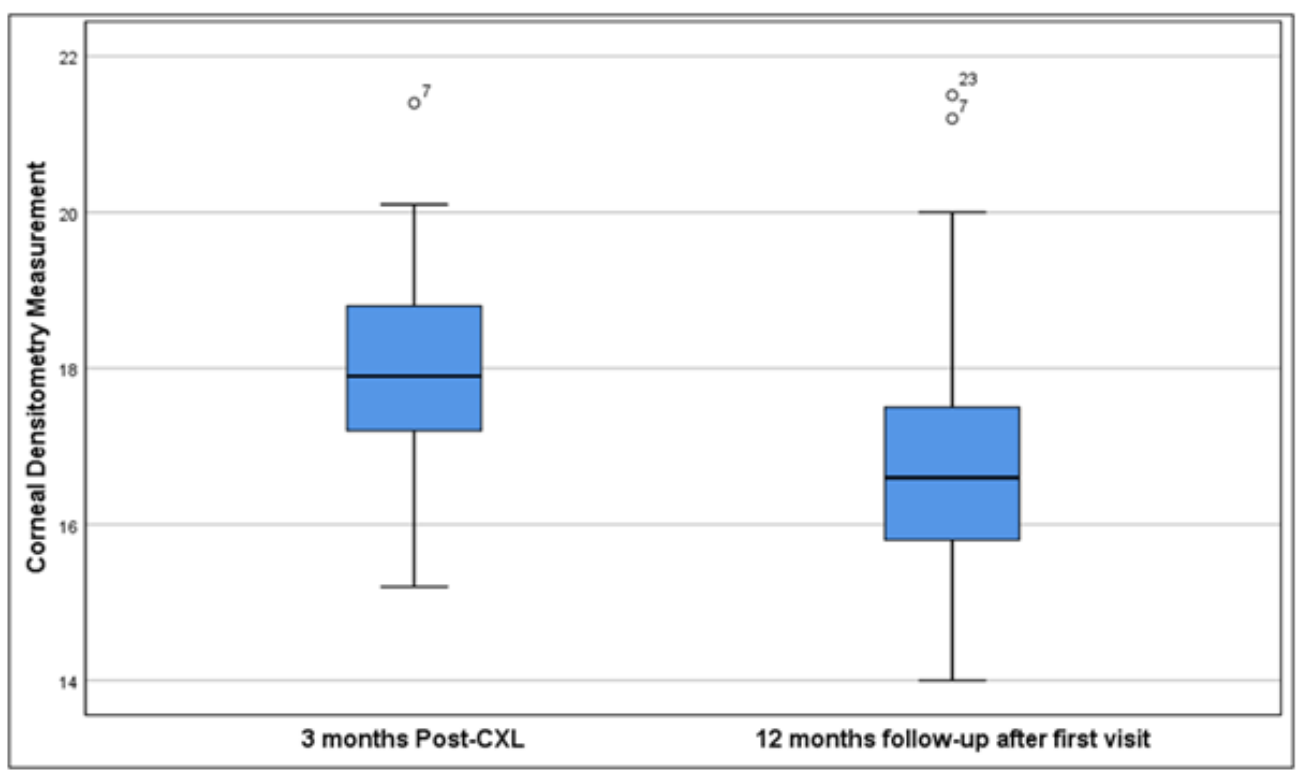


Figure 5

Corneal Densitometry measurements on two occasions post-crosslinking

\section{Group B}

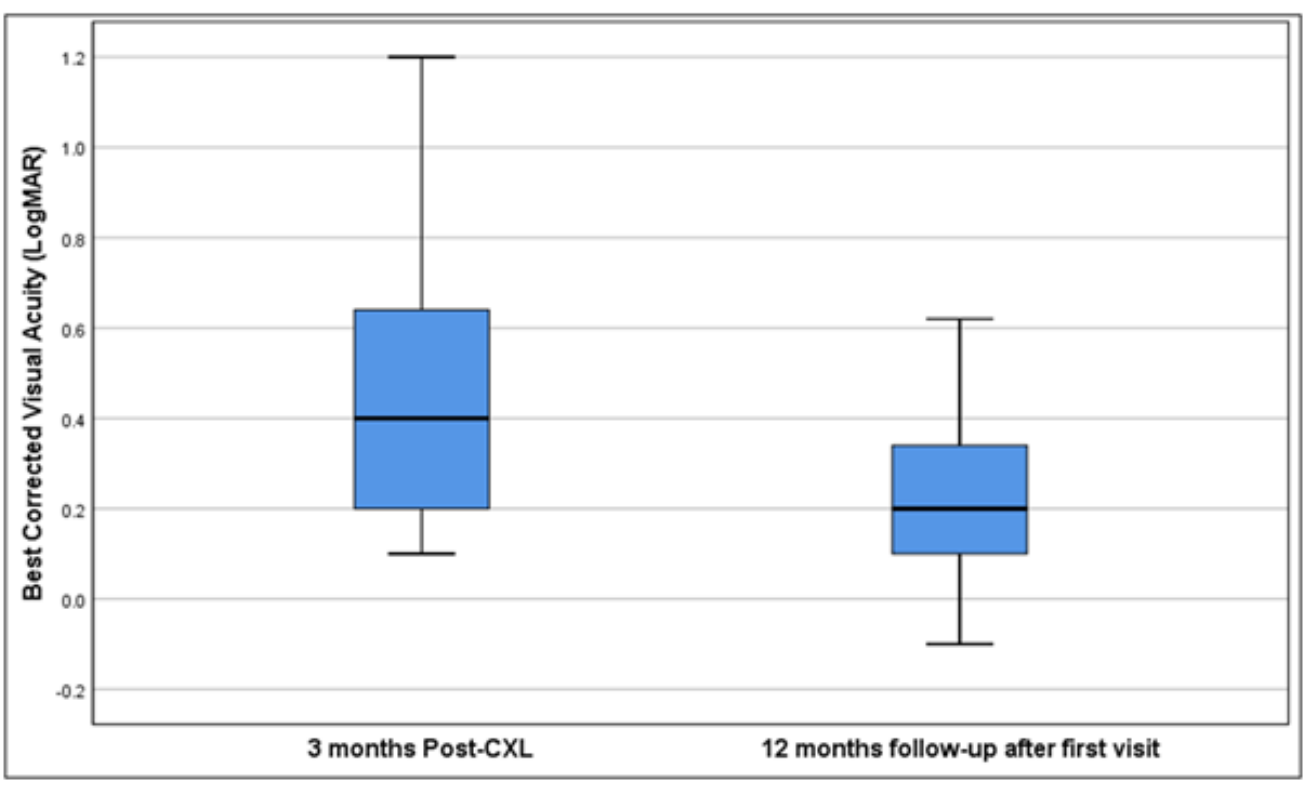

Figure 6

Post-crosslinking BCVA on two occasions

\section{Supplementary Files}

This is a list of supplementary files associated with this preprint. Click to download.

- Tables.pptx 